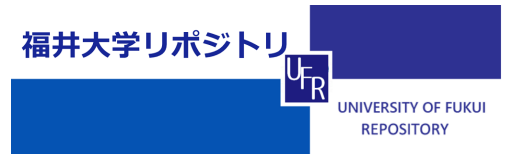
A Modi fi ed Met hod for Ci r cuit Anal ysi s usi ng Haar Wavel et Transf or $m$ wi th Adapt i ve Resol ut i on -For Ci rcui ts wi th Wavef or m wi th Shar $p$ Convex Ranges-

\begin{tabular}{|l|l|}
\hline 著者 & $\begin{array}{l}\text { O SH Nasanor i , MORO Sei i chi ro, MATSUMOTO } \\
\text { Tadashi }\end{array}$ \\
\hline $\begin{array}{l}\text { j our nal or } \\
\text { publ i cat i on t i t l e }\end{array}$ & $\begin{array}{l}\text { Pr oceedi ngs of Eur opean Conf er ence on Ci r cui t } \\
\text { Theor y and Desi gn ( ECCTD09) }\end{array}$ \\
\hline page range & $299-302$ \\
\hline year & $2009-08$ \\
\hline URL & ht t p: //hdl . handl e. net /10098/2351 \\
\hline
\end{tabular}




\section{A Modified Method for Circuit Analysis using Haar Wavelet Transform with Adaptive Resolution \\ -For Circuits with Waveform with Sharp Convex Ranges-}

\author{
Masanori Oishi and Seiichiro Moro \\ Department of Electrical and Electronics Engineering, \\ University of Fukui \\ 3-9-1 Bunkyo, Fukui, 910-8507 Japan \\ Email: \{oishi,moro\}@ppc8100.fuee.u-fukui.ac.jp
}

\author{
Tadashi Matsumoto \\ Department of Electrical and Electronics Engineering, \\ Fukui University of Technology \\ 3-6-1 Gakuen, Fukui, 910-8505 Japan \\ Email: matsu@ccmails.fukui-ut.ac.jp
}

\begin{abstract}
In this paper, we propose a modified method for the circuit analysis using wavelet transform with adaptive resolutions. Recently, many approaches to the circuit analysis using the wavelet transform have been proposed. Previously, we proposed method which can choose adaptive resolution automatically using multiresolution analysis. However, the method cannot be applied when the waveform has the sharp convex ranges. The proposed method improves the way to pick out the range and achieves more accurate and efficient calculations.
\end{abstract}

\section{INTRODUCTION}

The wavelet transform has been often used in signal processing because of its orthogonality and multiresolution property. Recently, much attention has been paid to the method for circuit analysis using wavelet transform [7]-[14]. In particular, Barmada et al. have proposed the Fourier-like approach for the circuit analysis using the wavelet transform [11]. In [11], the integral and differential operator matrices are introduced to the analysis, and the differential and integral equations are transformed into the algebraic equations like as using Fourier or Laplace transform. Moreover, the method can treat time varying or nonlinear circuits. Therefore, this method is useful for various circuit analyses.

However, in that method, the use of Daubechies wavelet makes the handling of the operator matrices complicated, especially, in the edges of the interval. Thus, we have proposed the circuit analysis method using Haar wavelet transform [14]. The Haar wavelet is easy to handle itself, and the operator matrices using the Haar wavelets are easily derived by introducing the block pulse functions [5], [6]. Moreover, the proposed method can treat the nonlinear time varying circuits.

In addition, Haar wavelets have the merit to be able to analyze the trajectory near the singular points where the trajectory moves rapidly with high resolution because of the orthogonality and localization property of the wavelet functions. As circuit analysis methods using this merit, some methods were proposed to pick out the ranges automatically where the trajectory moves rapidly near singular points. [16] However, these methods cannot be applied when the slope of a curve switches in a short time, because we considered only the first-derivative. To overcome this problem, it is considered that we can propose a better method if the way to pick out the range using second-derivative value and the previous way are combined. In this paper, we propose a modified method for the circuit analysis using wavelet transform with adaptive resolutions by automatically picking out the ranges which require higher resolution analysis by using both the result of the multiresolution analysis and the slope of derivative value. By the proposed method, it is considered that more accurate and efficient calculation can be achieved even if the behavior of the circuit is hardly predicted. In this paper, we confirm the effectiveness of the above method using a simple example.

\section{HAAR WAVELET MATRIX}

Haar functions are defined on interval $[0,1)$ as follows,

$$
\begin{aligned}
& h_{0}=\frac{1}{\sqrt{m}}, \\
& h_{i}=\frac{1}{\sqrt{m}} \times \begin{cases}2^{\frac{j}{2}}, & \frac{k-1}{2^{j}} \leq t<\frac{k-\frac{1}{2}}{2^{j}}, \\
-2^{\frac{j}{2}}, & \frac{k-\frac{1}{2}}{2^{j}} \leq t<\frac{k}{2^{j}}, \\
0, & \text { otherwise in }[0,1),\end{cases} \\
& i=0,1, \cdots, m-1, m=2^{\alpha},
\end{aligned}
$$

where $\alpha$ is positive integer, and $j$ and $k$ are nonnegative integers which satisfy $i=2^{j}+k$, i.e., $k=0,1, \cdots, 2^{j}-1$ for $j=0,1,2, \cdots$.

$\vec{y}$ is $m \times 1$-dimensional vector whose elements are the discretized expression of $y(t)$ and $\vec{c}$ is $m \times 1$-dimensional coefficient vector. $H$ is $m \times m$-dimensional Haar wavelet matrix defined as

$$
H=\left[\begin{array}{c}
\vec{h}_{0} \\
\vec{h}_{1} \\
\vdots \\
\vec{h}_{m-1}
\end{array}\right],
$$

where $\vec{h}_{i}$ is $1 \times m$-dimensional Haar wavelet basis vector whose elements are the discretized expression of $h_{i}(t)$. Using 
these vectors and matrix, Haar wavelet transform and inverse Haar wavelet transform are described as follows, respectively,

$$
\begin{gathered}
\vec{c}=H \vec{y}, \\
\vec{y}=H^{T} \vec{c}\left(=H^{-1} \vec{c}\right) .
\end{gathered}
$$

\section{INTEGRAL AND DifFERENTIAL OPERATOR MATRICES USING HAAR WAVELET}

The basic idea of the operator matrix has been firstly introduced by using Walsh function [5]. However, in logical way, the matrices introduced by block pulse function are more fundamental [4], [5]. The block pulse function is the set of $m$ rectangular pulses which have $1 / \mathrm{m}$ width and are shifted $1 / \mathrm{m}$ each other.

The integral operator matrix of the block pulse function matrix $B$ is defined as the following equation [5], [6].

$$
\begin{gathered}
\int_{0}^{i} B(\tau) d \tau \equiv Q_{B} \cdot B(t), \\
Q_{B(m \times m)}=\frac{1}{m}\left[\frac{1}{2} I_{(m \times m)}+\sum_{i=1}^{\infty} P_{(m \times m)}^{i}\right],
\end{gathered}
$$

where $B(t)$ is $m \times m$-dimensional matrix whose elements are the discretized expression of the block pulse functions $b_{i}(t)$, $i=0,1, \cdots, m-1$ and

$$
\begin{gathered}
P_{(m \times m)}^{i}=\left[\begin{array}{l|l}
0 & \mathrm{I}_{(m-i) \times(m-i)} \\
\hline 0_{(i \times i)} & 0
\end{array}\right] \text { for } i<m, \\
P_{(m \times m)}^{i}=0_{(m \times m)} \text { for } i \geq m .
\end{gathered}
$$

And the inverse matrix $Q_{B(m \times m)}^{-1}$ is calculated as follows [5]:

$$
Q_{B(m \times m)}^{-1}=4 m\left[\frac{1}{2} I_{(m \times m)}+\sum_{i=1}^{m-1}(-1)^{i} P_{(m \times m)}^{i}\right] .
$$

As the Haar wavelet matrix $H$ is the set of the orthogonal functions, the integral matrix of $H$ is given as follows:

$$
Q_{H}=H Q_{B}^{T} H^{-1}=H Q_{B}^{T} H^{T}
$$

Similarly, the differential matrix of $H$ can be written as

$$
Q_{H}^{-1}=H\left(Q_{B}^{T}\right)^{-1} H^{-1}=H\left(Q_{B}^{T}\right)^{-1} H^{T} .
$$

IV. HAAR WAVELET EXPRESSION OF BRANCH CHARACTERISTICS OF NONLINEAR TIME INVARIANT CirCuit ElEMENTS

As the Haar wavelet is defined on interval $[0,1)$, the generic interval $\left[t_{\min }, t_{\max }\right)$ can be rescaled by a new variable $\tau$ on $[0,1)$, where $\left[t_{\max }-t_{\min }\right) \tau+t_{\min }$. In this paper, $t_{\min }=$ 0 without loss of generality, then capacitance $c[\mathrm{~F}]$ and inductance $l[\mathrm{H}]$ are scaled to $C=c / t_{\max }$ and $L=l / t_{\max }$, respectively.

Next, we show the Haar wavelet expression of branch characteristics of nonlinear time varying circuit elements for

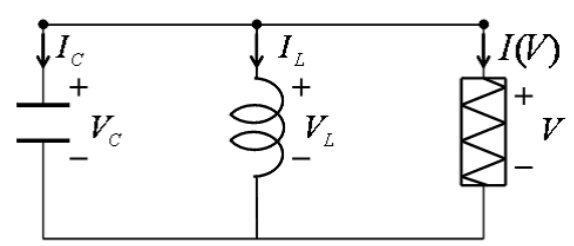

Fig. 1. Van der Pol oscillator circuit.

the expression in wavelet domain. See details in [14].

Capacitor:

$$
\begin{gathered}
V=C_{w}^{-1}\left(C_{0} V_{0}+Q_{H} I\right) \\
C_{w}=H \operatorname{diag}\left[C\left(i_{0}, t_{0}\right), C\left(i_{1}, t_{1}\right), \cdots, C\left(i_{m-1}, t_{m-1}\right)\right] H^{T}
\end{gathered}
$$

Inductor:

$$
\begin{gathered}
I=L_{w}^{-1}\left(L_{0} I_{0}+Q_{H} V\right) \\
L_{w}=H \operatorname{diag}\left[L\left(i_{0}, t_{0}\right), L\left(i_{1}, t_{1}\right), \cdots, L\left(i_{m-1}, t_{m-1}\right)\right] H^{T}
\end{gathered}
$$

Resistor:

$$
V=R_{w} I
$$

$$
R_{w}=H \operatorname{diag}\left[R\left(i_{0}, t_{0}\right), R\left(i_{1}, t_{1}\right), \cdots, R\left(i_{m-1}, t_{m-1}\right)\right] H^{T}
$$

\section{Circuit Analysis using Adaptive Resolution With Multiresolution AnAlysis [16]}

In this section, we show a previous method for automatically picking out the ranges to use the adaptive resolution by analyzing a example as follow.

The circuit model is Van der Pol oscillator circuit as shown in Fig. 1. The characteristic of nonlinear resistor of this circuit is;

$$
i(v)=-g_{1} v+g_{3} v^{3}=\left(-g_{1}+g_{3} v^{2}\right) v,
$$

and branch equations in the wavelet domain are shown as;

$$
\begin{aligned}
& I_{C}=C_{\omega} Q_{H}^{-1}\left(V-V_{0}\right), \\
& I_{L}=I_{L 0}+L_{\omega}^{-1} Q_{H} V, \\
& I(V)=G_{w} V .
\end{aligned}
$$

where $H \vec{v}\left(0_{-}\right)=V_{0}, H \vec{i}_{L}\left(0_{-}\right)=I_{L 0}, H \vec{v}=V$, $G_{w}=H \operatorname{diag}\left[-g_{1}+g_{3} v_{0}^{2},-g_{1}+g_{3} v_{1}^{2}, \cdots,-g_{1}+g_{3} v_{m-1}^{2}\right] H^{T}$. In this example, we take $g_{1}=2, g_{3}=1, C=0.05[\mathrm{~F}], L=$ $0.05[\mathrm{H}], v_{C}\left(0_{-}\right)=0[\mathrm{~V}], i_{L}\left(0_{-}\right)=1[\mathrm{~A}]$.

From these characteristics, the nonlinear algebraic equations are solved numerically with respect to $V$, and calculation result for $m=64$ without the adaptive resolution is shown in Fig. 2. If the waveforms have the singular points, more detailed analyses are needed by using the smaller intervals. However, it makes the calculation cost higher, for example, by taking $m=128$. Even if waveforms are predicted by experimentally imagine, we cannot predict the singular points for every circuit 


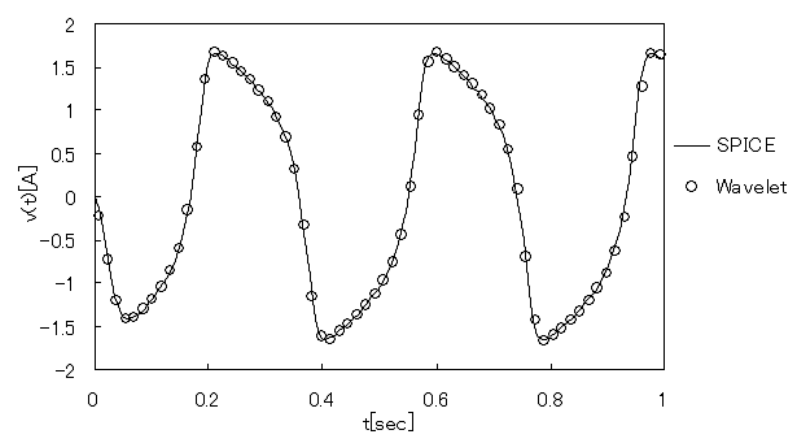

Fig. 2. Calculation result for $m=64$ without adaptive resolution [16].
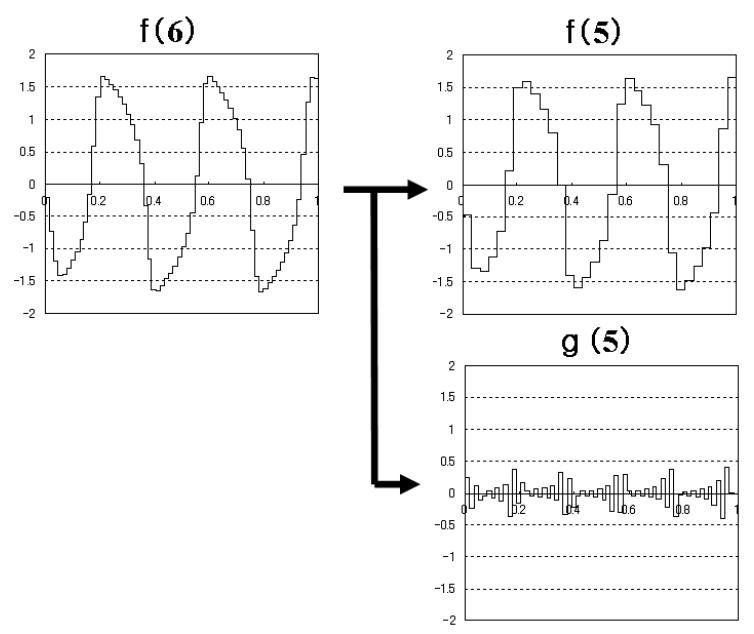

Fig. 3. Multiresolution analysis for $m=64$.

in the real world. To overcome this problem, we proposed the method using the results of the multiresolution analysis [16].

Figure 3 shows the results of the multiresolution analysis of the waveform shown in Fig. 2. $f(j)$ indicates the amounts of the results for $0,1, \cdots, j-1$-th resolution wavelets and $g(j)$ indicates the $j$-th resolution ones. Note that $f(6)=f(5)+$ $g(5)$. Because $g(j)$ can be considered as the error between the lower and the higher resolution cases, if $g(j)$ is large then the approximation is not sufficient. On the other hand, if $g(j)$ is small, it is considered that the approximation has already achieved. In this method, we take the threshold $\varepsilon$ that mean value of absolute value for $g(j)$, then pick out the ranges for $g(j)>\varepsilon$, and the thin lines indicate the threshold $\pm \varepsilon$ shown in fig 4 . In those ranges, we analyze the circuit again with higher

TABLE I

Picked OUt By MultiResolution.

\begin{tabular}{ccc}
\hline picked out range & total plots $p$ & sampling width \\
\hline $0-0.02$ & 2 & $1 / 4$ \\
$0.16-0.21$ & 4 & $1 / 8$ \\
$0.35-0.40$ & 4 & $1 / 8$ \\
$0.54-0.59$ & 4 & $1 / 8$ \\
$0.73-0.77$ & 4 & $1 / 8$ \\
$0.91-0.96$ & 4 & $1 / 8$ \\
\hline
\end{tabular}

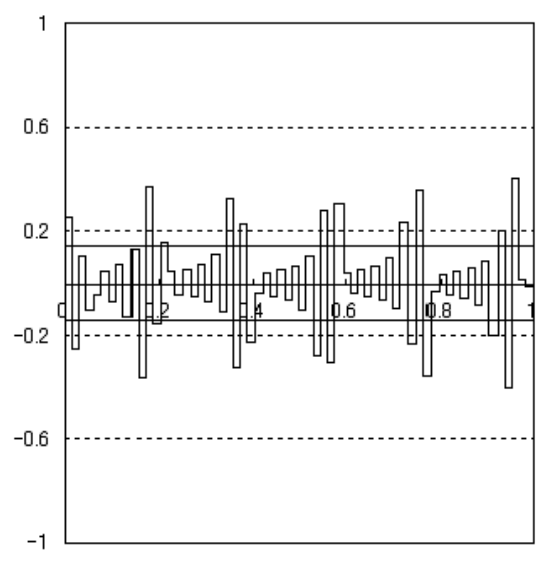

Fig. 4. Multiresolution analysis $\mathrm{g}(5)$.

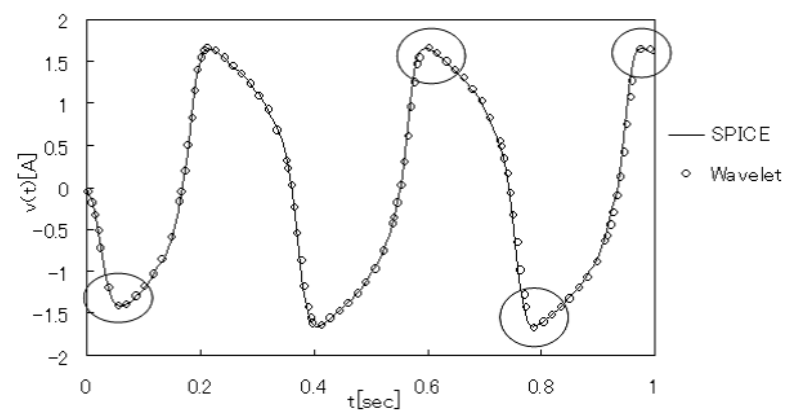

Fig. 5. Calculation result with adaptive resolution.

resolution wavelets. To avoid the over sampling, we set the sampling width as $1 / 2^{\alpha}$ to satisfy $2^{\alpha-1}<2 p \leq 2^{\alpha}$ where $p$ is the number of plots in the picked out ranges. Table I shows the relationship between the picked out ranges and the sampling width. The result for this method is shown in Fig. 5. The total number of plots is 97 and it is less than 128 that are the number of plots for the case of one higher resolution analysis. However, there is a problem that the method cannot achieve a good approximation when the slope of the curve switches in a short time. In the circled ranges of Fig. 5, it is necessary to be analyzed more precisely, because the waveform has sharp convex ranges in those ranges. In the next section, to overcome this problem, we propose the modified method.

TABLE II

PICKED OUt B y DeRIVATIVE OF FOR $m=64$.

\begin{tabular}{ccc}
\hline picked out range & total plots $p$ & sampling width \\
\hline $0.03-0.09$ & 4 & $1 / 8$ \\
$0.20-0.24$ & 4 & $1 / 8$ \\
$0.38-0.43$ & 4 & $1 / 8$ \\
$0.57-0.61$ & 4 & $1 / 8$ \\
$0.76-0.80$ & 4 & $1 / 8$ \\
$0.95-0.99$ & 4 & $1 / 8$ \\
\hline
\end{tabular}




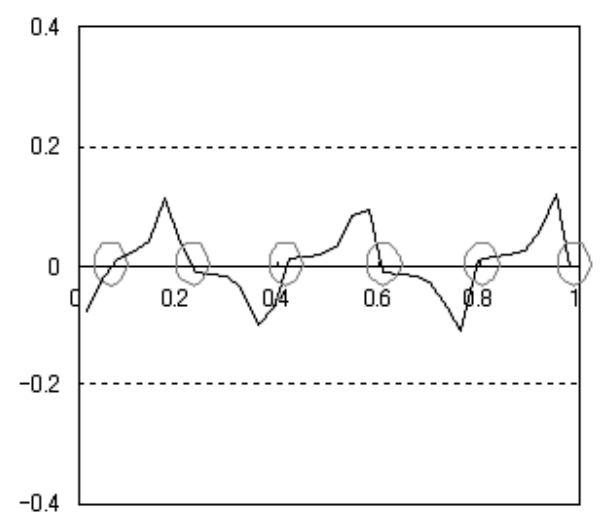

Fig. 6. Derivative of calculation result $(m=64)$ per difference between the two extreme values of calculation result.

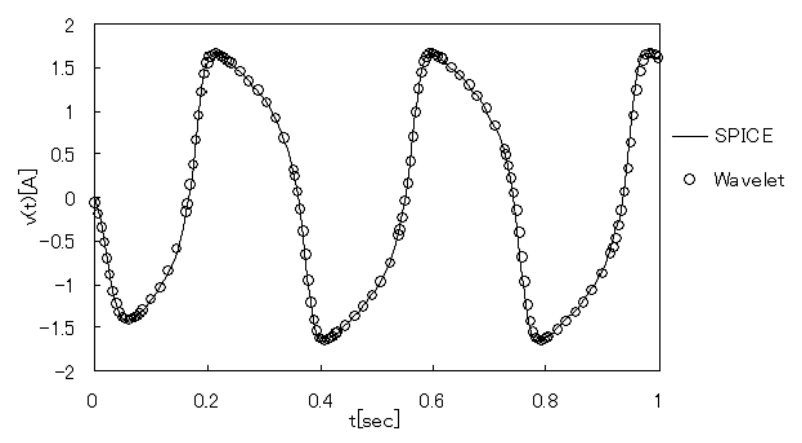

Fig. 7. Calculation result with modified method.

\section{IMPROVED METHOD FOR CiRCUIT ANALYSIS USING HAAR WAVELET}

In this section, to analyze sharp convex ranges, in addition to the method shown in [16], we pick out the ranges with high second-derivative value at zero crossing points of firstderivative value. Figure 6 shows the first-derivative of calculation result $(m=64)$ per difference between the two extreme values of calculation result. The slope of this curve at the zero crossing points are compared, and the ranges with large slope values are picked out and analyzed. In this example, we take the threshold equal $|0.3|$, and the ranges exceeding the threshold are picked out with four plots range for calculation result $(\mathrm{m}=64)$ centering around zero crossing point as shown in Table II. Calculation result is modified by combined two picking-out methods, and the relationship between the picked out ranges and the sampling width is shown in table III. Figure 7 shows the calculation result with modified method. The total number of plots is 134 and it is comparable with one higher resolution case, and this result is more accurate in sharp convex ranges. Applying this process recursively, it is considered that more accurate and efficient calculation can be achieved for circuit analysis.

\section{CONCLUSIONS}

In this paper, we have proposed a modified method for the circuit analysis using wavelet transform and second-derivative
TABLE III

Combined Two Pick Out Methods.

\begin{tabular}{ccc}
\hline picked out range & total plots $p$ & sampling width \\
\hline $0-0.09$ & 6 & $1 / 16$ \\
$0.16-0.24$ & 6 & $1 / 16$ \\
$0.35-0.43$ & 6 & $1 / 16$ \\
$0.54-0.61$ & 6 & $1 / 16$ \\
$0.73-0.80$ & 6 & $1 / 16$ \\
$0.91-0.99$ & 6 & $1 / 16$ \\
\hline
\end{tabular}

result with adaptive resolutions. In particular, the sharp convex ranges can be also picked out automatically. By the proposed method, it is considered that more accurate and efficient calculation can be achieved even if the behavior of the circuit is hardly predicted.

At this moment, the accuracy of the method is not enough discussed. The accuracy depends on the discussion how to pick out range with zero crossing point value is one of our future problems.

\section{REFERENCES}

[1] A. Haar, "Zur Theorie der orthogonalem funktionen System," Math. Ann., vol.69, pp.331-371, 1910.

[2] G. Strang, "Wavelet transforms versus Fourier Transforms," Bull. Amer. Math. Soc., vol.28, no.2, pp.288-305, Apr. 1993.

[3] C.F. Chen and C.H. Hsiao, "Haar wavelet method for solving lumped and distributed-parameter systems," IEE Proc. of Control Theory Appl., vol.144, no.1, pp.87-93, Jan. 1997.

[4] J.L. Wu, C.H. Chen, and C.F. Chen, "A model reduction via Haar wavelet," Int. J Control and Intelligent Systems, vol.29, no.2, pp. 2932, 2001.

[5] C.F. Chen, Y.T. Tsay, and T.T. Wu, "Walsh operational matrices for fractional calculus and their application to distributed systems," J. Franklin Institute, vol.303, no.3, pp.267-284, Mar. 1977.

[6] J.L. Wu, C.H. Chen, and C.F. Chen, "Numerical inversion of Laplace transform using Haar wavelet operational matrices," IEEE Trans. Circuits Syst. I, vol.48, no.1, pp.120-122, Jan. 2001.

[7] D. Zhou, X. Li, W. Zhang and W. Cai, "Nonlinear circuit simulation based on adaptive wavelet method," Proc. of IEEE International Symposium on Circuits and Systems (ISCAS'97), pp.1720-1723, June 1997.

[8] T. Matsumoto, T. Araki, and S. Moro, "Solving numerically stiff differential equations based on adaptive wavelet collocation methods," Proc. of IEEE International Symposium on Intelligent Signal Processing and Communication Systems (ISPACS'99), pp.669-672, Dec. 1999.

[9] D. Zhou and W. Cai, "A fast wavelet collocation method for high-speed circuit simulation," IEEE Trans. Circuits Syst. I, vol.46, no.8, pp.920-930, Aug. 1999.

[10] D. Zhou, W. Cai, and W. Zhang, "An adaptive wavelet method for nonlinear circuit simulation," IEEE Trans. Circuits Syst. I, vol.46, no.8, pp.931-938, Aug. 1999.

[11] S. Barmada and M. Raugi, "A general tool for circuit analysis based on wavelet transform,” Int. J Circuit Theory Appl., vol.28, no.5, pp.461-480, 2000

[12] X. Li, B. Hu, X. Ling, X. Zeng, "A wavelet-balance approach for steadystate analysis of nonlinear circuits," IEEE Trans. Circuits Syst. I, vol.49, no.5, pp.689-694, May 2002.

[13] G. Antonini and A. Orlandi, "A wavelet-based timedomain solution for PEEC circuit," IEEE Trans. Circuits Syst. I, vol.47, no.11, pp.1634-1639, Nov. 2000.

[14] A. Ohkubo, S. Moro, and T. Matsumoto, "A method for circuit analysis using Haar wavelet transforms," Proc. of IEEE Midwest Symposium on Circuits and Systems (MWSCAS'04), vol.3, pp.399-402, July 2004.

[15] Y. Nievergelt, Wavelets Made Easy, Birkhauser, 1999.

[16] M. Oishi, S. Moro, and T. Matsumoto, "A Method for Circuit Analysis using Haar Wavelet Transform with Adaptive Resolution," Proc. of International Symposium on Nonlinear Theory and its Applications (NOLTA'08), pp.369-372, Sep. 2008. 